\title{
Mare Indicum Temps et espaces d'une autre modernité
}

Mare Indicum Time and space for another modernity

Silvia M. Pizzetti

Traducteur : Silvia M. Pizzetti

\section{(2) OpenEdition \\ Journals}

\section{Édition électronique}

URL : https://journals.openedition.org/ahrf/13046

DOI : 10.4000/ahrf.13046

ISSN : $1952-403 X$

\section{Éditeur :}

Armand Colin, Société des études robespierristes

\section{Édition imprimée}

Date de publication : 1 mars 2014

Pagination : 9-26

ISBN : 978-2-200-9083-2790-8

ISSN : 0003-4436

\section{Référence électronique}

Silvia M. Pizzetti, « Mare Indicum Temps et espaces d'une autre modernité », Annales historiques de la Révolution française [En ligne], 375 | janvier-mars 2014, mis en ligne le 01 juillet 2017, consulté le 05 juillet 2021. URL : http://journals.openedition.org/ahrf/13046 ; DOI : https://doi.org/10.4000/ahrf. 13046 


\begin{abstract}
ARTICLES
MARE INDICUM TEMPS ET ESPACES D'UNE AUTRE MODERNITÉ
\end{abstract}

Silvia M. PIZZETTI

\begin{abstract}
Dans les dernières années, le corpus croissant d'études sur l'Océan Indien a soulevé bien des questions de méthodologie, mais aussi de nombreuses possibilités pour la recherche comparative. Le colonialisme a été un facteur évident pour donner unité à l'espace de l'Océan Indien. La religion était un autre domaine important de l'interaction. Les questions qui se posent quant à la nature et la dynamique de ces formes historiques de cohésion sont nombreux. Comment, par exemple, les migrations et les expériences de résistance anticoloniale ont façonné les subjectivités modernes dans l'Océan Indien? Comment pouvons-nous véritablement comprendre la notion de multiculturalisme dans l'Oéan Indien? Comment ont été forgées les identités dans l'Océan Indien, et quel rôle ont joué dans ce processus les réseaux religieux, commerciaux et culturels? De nouvelles études viennent de traiter certaines de ces questions en s'appuyant sur les conclusions émergeant du transnationalisme, l'universalisme et les régimes de circulation dans l'Océan Indien, en suggérant de nouveaux cadres théoriques pour comprendre et explorer de nouvelles méthodologies.
\end{abstract}

Mots-clés : Inde, Océan Indien, historiographie, colonialisme, postcolonialisme

Pendant les dernières vingt années, l'historiographie a abandonné la polarisation classique entre historical land et unhistorical sea, voyant donc les océans comme un lieu social spécifique, où des cultures différentes se 
rencontrent, s'affrontent, se fondent, suivant des relations soit asymétriques (colonisation, esclavagisme, etc.), soit de contamination et d'intégration ${ }^{1}$.

Le rôle des océans dans l'histoire moderne a été énorme, mais souvent contradictoire : c'est pour cette raison que l'histoire des océans a bénéficié, au cours de ces dernières années, d'un progrès significatif, dont témoigne la richesse de la production scientifique, par la variété des prises de contact et des interprétations, et par le caractère international des recherches. On peut donc dire qu'on a commencé à situer l'étude des océans dans un contexte historique.

\section{Les études sur l'Océan Indien : pour une nouvelle thalassologie}

Malgré quelques recherches anticipatrices conduites autour du milieu du siècle dernier, on peut dire qu'un domaine d'étude spécifique sur l'Océan Indien a commencé à s'affirmer seulement après $1980^{2}$ : à ce propos, ont joué un rôle fondamental d'un côté la diffusion du paradigme des Annales (en particulier la réflexion géo-historique sur la Méditerranée conduite par Fernand Braudel) et, de l'autre, l'affirmation, aux États-Unis, du modèle d'analyse d'Immanuel Wallerstein sur le système-monde.

Du point de vue d'une nouvelle World History, beaucoup de chercheurs occidentaux ont commencé à regarder l'Océan Indien pour y trouver une sorte de parallélisme avec la Méditerranée, en individualisant de petites Méditerranées qui peuvent être considérées comme des unités d'étude 3 . Les historiens, ainsi, ont identifié une "Méditerranée chinoise », une "Méditerranée japonaise », une « Méditerranée du Sud-est Asiatique » (qui comprend les Philippines), une «Méditerranée indienne » et une « Méditerranée de langue arabe », pour ne parler que des principales d'entre elles. Est en train de s'imposer, donc, une perspective méditerranéenne (« Mediterranean thinking ») qui considère les mers et les océans selon une analyse historique qui part du structuralisme géo-historique, suivant

(1) À ce propos, voir les intéressants travaux de Jean-Loup AMSELLE, Logiques métisses. Anthropologie de l'identité en Afrique et ailleurs, Paris, Payot, 1990 ; James CLIFFORD, Roots. Travel and Translation in the Late Twentieth Century, London, Harvard University Press, 1997 ; Serge GRUZINSKI, Les quatre parties du monde. Histoire d'une mondialisation, Paris, La Martinière, 2004.

(2) Voir Alan VILLIERS, The Indian Ocean, London, Museum Press, 1952 ; Id., Monsoon Seas. The Story of the Indian Ocean, New York, McGraw-Hill, 1952 ; Mauritius Auguste TOUSSAINT, Histoire de l'Océan Indien, Paris, PUF, 1980. Plus récemment, voir aussi Jean-Louis GuEBOURG, Petites îles et archipels de l'Océan Indien, Paris, Karthala, 1999.

(3) Voir Kären WIGEN, « Introduction », American Historical Review, 111/3, June 2006, p. 718 ; "Peregine HORDEN, Nicholas PuRCELL, «The "Mediterranean" and the new thalassology », American Historical Review, 111/3, Juin 2006, p. 723. 
un modèle d'histoire «totale » qui développe sa reconstruction temporelle à partir d'une tripartition en structures, conjonctures et événements.

C'est pour cela qu'au cours de ces trente dernières années ${ }^{4}$, d'importants ouvrages généraux ont commencé à paraître suivant cette nouvelle façon de voir : il suffit de mentionner, à ce propos, les travaux de Chaudhuri $(1985,1990)^{5}$, Chandra (1987) ${ }^{6}$, Das Gupta-Pearson (1987) ${ }^{7}$, LombardAubin $(1988,2000)^{8}$, McPherson $(1993)^{9}$, Hall (1996) ${ }^{10}$, MukherjeeSubramanian $(1999)^{11}$, Dasgupta (2001) $)^{12}$, Kearney (2003) $)^{13}$, Pearson (2003, $2005)^{14}$, Bose $(2006)^{15}$ et Alpers-Ray $(2008)^{16}$. À partir des années $80 \mathrm{du}$ siècle dernier trois chercheurs - Kirti N. Chaudhuri, Michael Pearson et Kenneth McPherson - se sont imposés avec autorité comme représentatifs de cette nouvelle thalassologie, en influençant d'une manière durable toutes les recherches suivantes.

(4) Pour une première introduction à l'historiographie sur l'Océan Indien et sur ses résultats, voir Sinnappah ARASARATNAM, « Recent Trends in the Historiography of the Indian Ocean, 1500$1800 »$, Journal of World History, 1/2, 1990, p. 225-48 ; John WILLS Jr, « Maritime Asia, 1500-1800 : the interactive Emergence of European Domination », American Historical Review, 98/1, 1993, p. 83-105 ; Markus VINK, « From Port-city to World-System. Spatial Constructs in Dutch Indian Ocean Studies, 1500-1800», Itinerario, 28/2, 2004, p. 45-116 ; Markus VINK, « Indian Ocean Studies and the "new Thalassology" », Journal of Global History, 2, 2007, p. 42-62.

(5) Kirti N. Chaudhuri, Trade and Civilisation in the Indian Ocean. History from the Rise of Islam to 1750, Cambridge, Cambridge University Press, 1985 ; Kirti N. CHAUDHURI, Asia before Europe. Economy and civilization of the Indian Ocean from the Rise of Islam to 1750, Cambridge, Cambridge University Press, 1990.

(6) Satish CHANDRA (dir.), The Indian Ocean. Explorations in History, Commerce and Politics, New Delhi-Newbury Park, Sage Publications, 1987.

(7) Ashin DAS GUPTA, Michael PEARSON (dir.), India and the Indian Ocean, 1500-1800, Calcutta-New York, Oxford University Press, 1987.

(8) Denys LOMBARD, Jean AUBIN (dir.), Marchands et hommes d'affaires asiatiques dans l'Océan Indien et la Mer de Chine, 13e-20 siècles, Paris, EHESS, 1988 (édition en anglais, Asian Merchants and Businessmen in the Indian Ocean and the China Sea, New Delhi-New York, Oxford University Press, 2000).

(9) Kenneth McPherson, The Indian Ocean. A History of People and the Sea, New Delhi-New York, Oxford University Press, 1993.

(10) Richard HALl, Empires of the Monsoon. A history of the Indian Ocean and its Invaders, London, HarperCollins, 1996.

(11) Rudrangshu MuKHERJEe, Lakshmi Subramaniam (dir.), Politics and Trade in the Indian Ocean. Essays in honour of Ashin Das Gupta, New Delhi-Oxford, Oxford University Press, 1999.

(12) Uma DAS GUPTA (dir.), The world of the Indian Ocean Merchant 1500-1800. Collected Essays of Ashin Das Gupta, New Delhi-New York, Oxford University Press, 2001.

(13) Milo KEARNEY, The Indian Ocean in World History, London, Routledge, 2003.

(14) Michael PeARson, The Indian Ocean, London-New York, Routledge, 2003 ; Michael PEARSON, The world of the Indian Ocean, 1500-1800. Studies in Economic, Social and Cultural History, Burlington, Ashgate, 2005.

(15) Sugata BOSE, A hundred horizons. The Indian Ocean in the Age of Global Empire, Cambridge, Harvard University Press, 2006.

(16) Edward AlPERS, Himanshu RAY (dir.), Cross Currents and Community Networks. The History of the Indian Ocean World, New Delhi, Oxford University Press, 2008. 
Dans ses deux principales œuvres sur l'économie-monde dans l'Océan Indien, Kirti N. Chaudhuri reconstruit l'entrelacement complexe des trafics commerciaux sur la longue distance - où il voit déjà les fondements du capitalisme - qui caractérise cette zone géopolitique comme une seule unité historique, au-delà des divisions géographiques et culturelles.

Chaudhuri, qui est redevable à Braudel et moins à Wallerstein, voit du VII ${ }^{\mathrm{e}}$ à la moitié du XVIII ${ }^{\mathrm{e}}$ siècle - quatre forces prédominantes dans l'Océan Indien, qui sont : l'expansion de l'Islam du Proche-Orient à toute l'Asie du Sud, la présence massive et l'influence de la civilisation chinoise, les migrations périodiques des peuples nomades de l'Asie centrale, et, à partir du XVI ${ }^{\mathrm{e}}$ siècle, l'expansion maritime européenne. Pour Chaudhuri l'Océan Indien est caractérisé par une unité spécifique, même si les peuples, et les cultures ne sont pas soumis à un destin commun. Dans une analyse désenchantée qui reconnaît la présence inévitable de plusieurs éléments qui jouent contre une unité complète, l'Océan Indien représente de toutes façons une zone géopolitique intégrée ${ }^{17}$.

D'une manière analogue, Michael Pearson, suivant l'exemple de Braudel, a étudié la structure profonde qui peut garantir quelques éléments de continuité parmi les sociétés qui peuplent les côtes de l'Océan Indien. Il souligne cette considérable unité et continuité du point de vue de la géographie, de la topographie et des vents (les moussons) : les éléments structurels de l'océan - une situation qui n'a pas d'égal dans le monde favorisent et en même temps conditionnent la circulation des hommes, des marchandises et des idées ${ }^{18}$. En incluant dans son projet les peuples et la mer, Pearson reconnaît d'autres éléments d'unité dans le panorama culturel de la région : les villes portuaires, les navires et les matelots ; les trafics des marchandises ; le prosélytisme des différentes religions ; la naissance

(17) Kirti N. CHAUDHURI « Means of travel, movements of peoples, economic exchange, climate, and historical forces created elements of cohesion. Religion, social systems, and cultural traditions, on the other hand, provided the contrasts »: Trade and civilisation, p. 21 ; du même auteur : Asia before Europe, p. 104 et p. 383-7, et encore « The unity and Disunity of Indian Ocean History from the Rise of Islam to 1750 : the Outline of a Theory and Historical Discourse », Journal of World History, 4/1, 1993, p. 1 et p. 7.

(18) À ce propos, voir aussi Felipe FERnÁNDEZ-ARMESTO, «The Indian Ocean in World History », dans Anthony Disney, Emily BOOTH (dir.), Vasco da Gama and the Linking of Europe and Asia, New Delhi-New York, Oxford University Press, 2000, p. 16. 
d'une lingua franca ; et, finalement, au début de l'âge moderne, la présence portugaise sur les côtes de l'Asie du sud ${ }^{19}$.

Kenneth McPherson a également construit un modèle qui voit un ensemble de zones culturelles interconnectées, qui expliquent bien la richesse des échanges dans le premier centre d'activité commerciale et maritime du monde. McPherson, qui reconnait sa dette envers les études de Braudel, va dessiner un système-monde typique de l'Océan Indien, caractérisé par une identité régionale née de la relation entre le commerce maritime et un processus de diffusion culturelle, qui aboutissent à un monde interconnecté aux grandes routes de l'océan. Suivant Chaudhuri et Pearson, donc, McPherson ne considère pas l'Océan Indien comme une zone caractérisée par une civilisation unique ; toutefois il souligne que, à cause des nombreuses interactions culturelles qui dérivent de la singulière intensité des trafics, les peuples de l'Océan Indien ont certains traits culturels qui les distinguent des peuples des mondes voisins ${ }^{20}$.

Pour continuer à nous fonder sur l'analogie avec la Méditerranée employée par cette nouvelle thalassologie, les différentes «subMéditerranées » comprises dans le grand Océan ont reçu l'attention croissante des historiens pendant ces trente dernières années : il est suffisant de citer les travaux de Martin-Martin (1978) et de Barendse (2002) sur les mers d'Arabie ${ }^{21}$, ou bien de Subrahmanyam (1990), Gommans (1995), McPherson (1998) et Prakash-Lombard (1999) sur le Golfe du Bengale ${ }^{22}$; de Steinberg (et alii, 1987), Lombard (1990), Tarling (1992), Reid (1988-93; 1993 ; 2000), Wolters (1999), Osborne (2000), Lieberman (2003), Owen (2005) et Sardesai (2009) sur les mers du Sud-est Asiatique ${ }^{23}$; et, finalement, les œuvres de Wills (1979), Pan

(19) Michael PEARSON, « Introduction I : The state of the Subject », dans Uma DAS GUPTA, Michael PEARSON, India and the Indian Ocean, p. 12-17; Michael PEARSON, Indian Ocean, op. cit., p. 3-5.

(20) Kenneth MCPHERSON, « Cultural Exchange in the Indian Ocean Region », Westerly, 29/4, 1984, p. 5-16 ; Id., « Processes of Cultural Interaction in the Indian Ocean : An historical perspective », The Great Circle, 6/2, 1984, p. 78-92 ; Michael PEARSON, Indian Ocean, op. cit., p. 2-4 et p. 15.

(21) Esmond MARTIN, Chryssee MARTIN, Cargoes of the East. The ports, trade and culture of the Arabian Seas and western Indian Ocean, London, Elm Tree Books, 1978 ; René BARENDSE, The Arabian Seas. The Indian Ocean World of the Seventeenth Century, Armonk, M.E. Sharpe, 2002.

(22) Sanjay SubRAHMANYAM, The Political Economy of Commerce. Southern India, 15001650, Cambridge, Cambridge University Press, 1990 ; Jos GomMANS, « Trade and Civilization around the Bay of Bengal, c. 1650-1800», Itinerario, 19/3, 1995, p. 82-108; Kenneth MCPHERSON, «Trade and Traders in the Bay of Bengal. Fifteenth to Nineteenth Centuries», dans MUKHERJEE, SuBRAMANIAN, Politics and trade, p. 183-209 ; Om PRAKASH, Denys LOMBARD (dir.), Commerce and Culture in the Bay of Bengal, 1500-1800, New Delhi, Manohar, 1999.

(23) Joel SteInBERG(et alii), In search of Southeast Asia: a Modern history, Honolulu, University of Hawaii Press, 1987 (2e éd.); Denys LOMBARD, Le carrefour javanais. Essai d'histoire globale, 3 vol., Paris, EHESS, 1990 ; Nicholas TARLING (dir.), The Cambridge History of Southeast 
(1990), Wang (1991 ; 2000), Reid (1996), Blussé (1999), Hamilton (1999) et Hong Liu (2006) sur les mers de la Chine ${ }^{24}$.

Les modèles interprétatifs de la nouvelle thalassologie, bien sûr, ont suscité aussi des critiques radicales : Pierre Chaunu, par exemple, a souligné l'erreur de perspective qui se trouve au fond de tous les discours sur l'unité de l'espace économique et géopolitique indien, à son avis « rien d'autre qu'une extension de la Méditerranée orientale $»^{25}$. Niels Steensgaard, de son côté, décrit l'Océan Indien comme un système à l'état d'embryon, fort différent, du point de vue de la cohésion, tant de la Méditerranée, que de la Baltique et de l'Archipel Indonésien ; dans ses recherches sur le commerce inter-asiatique, il estime les trafics sur l'océan marginaux par rapport à l'ensemble de l'économie régionale ${ }^{26}$.

Peregrine Horden et Nicholas Purcell partagent quelques critiques et quelques doutes sur la nouvelle thalassologie : par rapport à une interdépendance écologique très étroite en Méditerranée, tous les espaces océaniques, y compris celui de l'Océan Indien, étaient caractérisés - et

Asia (vol. I: From early Times to c. 1800), New York, Cambridge University Press, 1992 ; Anthony REID, Southeast Asia in the Age of Commerce, 2 vol., New Haven, Yale University Press, 1988-1993; Anthony REID (dir.), Southeast Asia in the Early Modern Era. Trade, Power, and Belief, Ithaca, Cornell University Press, 1993 ; Anthony REID, Charting the Shape of Early Modern Southeast Asia, Singapore, Singapore University Press, 2000 ; O.W. WOLTERS, History, Culture, and Region in Southeast Asian Perspectives, Ithaca, Cornell University, 1999 (2e éd.) ; Milton OsBORNE, Southeast Asia. An introductory history, Crows Nest, Allen \& Unwin, 2000 ; Victor LIEBRMAN, Strange parallels (vol. I: Integration of the Mainland Southeast Asia in global Context, c. 800-1830), New York, Cambridge University Press, 2003 ; Norman OwEN (dir.), The Emergence of Modern Southeast Asia. A New History, Honolulu, University of Hawaii Press, 2005 ; D. R. SADERSAI, Southeast Asia. Past and Present, Boulder, Westview Press, 2009 (9e éd.).

(24) John E. WILLS Jr, «Maritime China from Wang Chih to Shih Lang: Themes in Peripheral History», dans Jonathan SPENCE, John E. WILLS Jr (dir.), From Ming to Ch'ing : Conquest, Region and Continuity in Seventeenth-Century China, New Haven, Yale University Press, 1979 ; Lynn PAN, Sons of the Yellow Emperor. The Story of the Overseas Chinese, Boston, Little-Brown, 1990 ; Gungwu Wang, China and the Chinese Overseas, Singapore, Times Academic Press, 1991 ; Gungwu WANG, The Chinese Overseas. From Earthbound China to the Quest for Autonomy, Cambridge, Harvard University Press, 2000 ; Anthony REID, Sojourners and Settlers. Histories of Southeast Asia and the Chinese, St. Leonards, Allen \& Unwin, 1996 ; Leonard BLussé, «Chinese Century. The Eighteenth Century in the China Sea Region», dans Archipel, 58/3, 1999, p. 107-29 ; Gary HAMILTON (dir.), Cosmopolitan Capitalists : Hong Kong and the Chinese Diaspora at the End of the Twentieth Century, Seattle, University of Washington Press, 1999 ; Hong LIU (dir.), The Chinese Overseas, 4 vol., London-New York, Routledge, 2006.

(25) Pierre Chaunu, European Expansion in the Later Middle Ages, Amsterdam, North Holland Publ. Co., 1979, p. 218-219.

(26) Niels STEENSGAARD, «The Indian Ocean Network and the Emerging World-Economy, c. 1500-1750», dans Satish CHANDRA, The Indian Ocean, op. cit. p. 127-8, p. 138 et p. 149. De la même opinion, Heather SUTHERLAND, « Southeast Asian History and the Mediterranean Analogy », dans Journal of Southeast Asian Studies, 34/1, 2003, p. 1-20. Pour la réponse de Pearson, voir Indian Ocean, op. cit., p. 5-6. 
limités - par des trafics d'élite plutôt que par des échanges basés sur les produits les plus communs ${ }^{27}$.

Aux côtés du Mediterranean thinking inspiré par Braudel, on peut voir de toute façon un autre axe d'étude, influencé par l'analyse systémique d'Immanuel Wallerstein ${ }^{28}$.

Wallerstein, qui reconnaît l'impossibilité de reconstruire d'une manière persuasive un cadre systémique général concernant l'Océan Indien, souligne toutefois que cette zone, au moins jusqu' au XIX ${ }^{\mathrm{e}}$ siècle, n'a pas connu des relations continuelles avec la première économie-monde, celle de l'Europe. En effet, il y eut seulement des échanges épisodiques basés presque uniquement sur les produits de luxe, les seuls vraiment importants pour tous, Européens et Asiatiques. Même si quelques-uns de ces produits de luxe - tissus, soieries, porcelaines, thé, café, épices, etc. - ont été exportés vers l'Europe en quantités croissantes à partir du XVII ${ }^{\mathrm{e}}$ siècle, pour Wallerstein, l'Asie n'a pas encore connu, à cette époque, une réelle intégration dans le système-monde capitaliste d'Europe, et elle n'est pas encore devenue sa «périphérie ».

Entre 1750 et 1850 le subcontinent indien et d'autres régions d'Asie ont été progressivement pris dans l'économie-monde européenne et ont subi une réorganisation des structures productives sous l'influence convergente du colonialisme et du capitalisme industriel. Un processus de désindustrialisation et de ré-féodalisation de l'économie - en particulier indienne va donc commencer, caractérisé par le développement des cultures d'exportation (indigo, soie écrue, opium et coton), par un nouveau système d'organisation foncière (un nouveau rôle pour les zamindari et les ryotwari), par les dettes provoquées par la diffusion du système des agences et des avances, et finalement par l'imposition du commerce triangulaire Inde-Chine-Grande Bretagne ${ }^{29}$.

(27) Peregrine HoRden et Nicholas PURCELL, « The "Mediterranean" and the new thalasso$\operatorname{logy} »$, p. 732-733 et p. 739. En particulier pour les critiques à Chaudhuri et à Pearson, voir p. 738-739, note 74 .

(28) Immanuel WallersteIn, World-Systems Analysis. An Introduction, Durham, Duke University Press, 2004 ; Id., The Essential Wallerstein, New York, New Press, 2000 ; Terence HoPKINS, Id., World-systems Analysis : Theory and Methodology, Beverly Hills, Sage Publications, 1982.

(29) Immanuel WALLERSTEIN, The Modern World System, 3 vol., New York, Academic Press, 1974-1989, vol. I, p. 39-46, p. 301-302, et p. 346-357 ; vol. II, p. 47, p. 50, et p. 273-274 ; vol. III, p. 16; $I d$., «The Incorporation of the Indian Subcontinent into the Capitalist World Economy», dans Satish CHANDRA, The Indian Ocean, p. 222-253 ; Id., «World-Systems Analysis and Historical Particularity : Some Comments», dans Sugata Bose, Ayesha Jalal, South Asia, History, Culture, Political Economy, Routledge, 2011, p. 21-26. 
Les chercheurs ont diversement répliqué à l'universalisme hégémonique qui est implicite dans le système-monde de Wallerstein ${ }^{30}$ : André Gunder Frank, Barry Gills, Samuel Adshead et Janet Abu-Lughod ont condamné ouvertement son point de vue euro-centrique, en affirmant que l'oecoumène Afro-Euroasiatique était déjà lié dans un seul réseau de marchés et d'échanges bien avant le moment où l'expansion européenne outremer a commencé ${ }^{31}$.

De leur côté les historiens globaux de la sino-centrique California School, par exemple Ken Pomeranz, Roy Bin Wong, Jack Goldstone et Richard Von Glahn, ont soutenu que, au moins jusqu'au moment où les intérêts impériaux et économiques liés au processus d'industrialisation ont eu de l'influence dans l'Angleterre du XIX' ${ }^{\mathrm{e}}$ siècle, un système-monde polycentrique - sans aucun centre de pouvoir dominant - a été prédominant ${ }^{32}$.

D'une manière analogue à la California School, d'autres chercheurs ont justement souligné qu' avant la fin XVII et le début du XVIII ${ }^{\mathrm{e}}$ siècle, les routes traditionnelles de communication commerciale par terre et par mer ont continué à se montrer vitales face au défi naval, politique et économique de l'Occident ${ }^{33}$.

Les partisans de la théorie du système-monde moderne (y compris le dernier Braudel) ont répliqué à ces critiques en soulignant qu'entre le $\mathrm{XVI}^{\mathrm{e}}$ et le $\mathrm{XIX}^{\mathrm{e}}$ siècle les trois économies-monde existantes en Asie

(30) Sur la partie européenne du système-monde moderne et sur le débat qui s’y rapporte, voir Maurice AYMARD (dir.), Dutch Capitalism and World Capitalism, Cambridge-New York, Cambridge University Press, 1982.

(31) André Gunder FranK, Barry GILLS, « World System Cycles, Crises, and Hegemonial Shifts, 1700 BC to 1700 AD », dans André GundER FRANK, Barry GILls (dir.), The World System. Five Hundred Years or five Thousand ?, London-New York Routledge, 1993, p. 143-199 (article déjà paru dans Review, 15/4, 1992, p. 621-687) ; Samuel ADSHEAD, Central Asia in World History, London, Macmillan, 1993 ; Janet ABU-Lughod, Before European Hegemony. The World System A.D. 1250-1350, New York, Oxford University Press, 1989.

(32) Voir Kenneth Pomeranz, The Great Divergence. China, Europe, and the Making of the Modern World Economy, Princeton, Princeton University Press, 2000 ; R. BIN Wong, China transformed. Historical Change and the Limits of European Experience, Ithaca, Cornell University Press, 1997 ; Roy BIN WONG, « The Search for European Differences and Domination in the Early Modern World. A View from Asia », American Historical Review, 107/2, 2002, p. 447-469.

(33) Voir Stephen DALE, Indian Merchants and Eurasian Trade, 1600-1750, Cambridge-New York, Cambridge University Press, 1994 ; Id., « Indo-Russian Trade in the Eighteenth Century », Sugata Bose, Ayesha Jalal, South Asia, p. 140-158 ; René BARENDSE, «Trade and State in the Arabian Seas : a Survey from the Fifteenth to the Eighteenth century », Journal of World History, 11/2, 2000, p. 183-189 et p. 193. À propos de la décadence des routes traditionnelles de communication entre Europe et Asie à partir du début du XVII ${ }^{\mathrm{e}}$ siècle, voir aussi des opinions tout à fait différentes : Om PRAKASH, European commercial Enterprise in Pre-colonial India, Cambridge, Cambridge University Press, 1998, p. 65 et p. 72 ; Niels STEENSGAARD, The Asian Trade Revolution of the Seventeenth Century. The East India Companies and the Decline of the Caravan Trade, Chicago, University of Chicago Press, 1973, p. 171-172 ; Thomas R. METCALF, Imperial Connections : India in the Indian Ocean Arena, 1860-1920, Berkeley, University of California Press, 2007, p. 184-189. 
(c'est-à-dire l'Islam, l'Inde et la Chine) étaient désormais « encerclées » par l'économie-monde européenne. C'est pour cela que l'Océan Indien, considéré dans son ensemble, faisait partie en réalité d'une énorme, mais fragile, super-économie-monde ${ }^{34}$. Braudel, en effet, introduisait une distinction fondamentale entre une économie d'échanges normale et traditionnelle, et une économie capitaliste et mercantile bien plus raffinée, née entre le $\mathrm{XV}^{\mathrm{e}}$ et le $\mathrm{XIX}^{\mathrm{e}}$ siècles. De plus, Braudel était convaincu de réussir à identifier quatre domaines d'interconnexion sociale - l'économique, la politique, la civilité matérielle et la hiérarchie sociale au lieu du simple déterminisme économique employé par Wallerstein ${ }^{35}$.

En complément de ces perspectives globalisantes, suivant le modèle d'une nouvelle World History, on a contesté aussi la définition de Wallerstein sur l'Océan Indien vu comme une arène extérieure car elle serait fondée sur une interprétation réductive des soi-disant produits de luxe : il faut donc considérer non seulement la qualité des produits, mais aussi le volume des échanges et le profit ainsi que le prestige international qui dérive du commerce d'Asie ${ }^{36}$.

Une quatrième position est celle qui caractérise les historiens indomusulmans (par exemple l'Aligarh School) ${ }^{37}$ : dans ce cas, on voit un système-monde de l'Océan Indien autonome ou bien une économie-monde ainsi que islamique fondée sur l'Inde et en particulier sur le Gujarat islamisé, dans laquelle les Européens ont pénétré à partir du XVI siècle sans toutefois réussir à l'assimiler.

Dans ce contexte interprétatif, l'Inde s'assure donc un rôle fondamental et stratégique, grâce à sa position médiane entre Europe et Asie (Orientale et du Sud-est) et à la richesse de son marché. Certains auteurs en sont arrivés à se demander - dans le rapport Europe-Asie - où se

(34) Fernand BRAUDEL, Civilisation matérielle, économie et capitalisme (XVe-XVIII siècle), 3 vol., Paris, Colin, 1979, vol III (Le temps du monde), p. 542. Sur l'interprétation de Braudel voir p. $505-542$.

(35) Ibidem, vol. II (Les jeux de l'échange), p. 21-22; vol. III, p. 26-69. Voir aussi la distinction entre économie de marché et économie-monde chez Kirti N. CHAUDHURI, «The World System East of Longitude 20 : the European Role in Asia, 1500-1750», dans Review, 5/2, 1981, p. 226 ; Jan KIENEWICZ, «Contact and Transformation : The European Pre-colonial Expansion in the Indian Ocean World-System in the 16th-17th centuries », Itinerario, 8/2, 1984, p. 45-46.

(36) Voir Michael PEARSON, Before Colonialism. Theories on Asian-European Relations, 1500-1750, New Delhi-New York, Oxford University Press, 1988, p. 9, p. 13, p. 17, p. 21, p. 34-36, p. 38-39, p. 46, p. 50, et p. 67-68 ; René J. BARENDSE, Trade and State, op. cit., p. 189-190 ; Kenneth MCPHERSON, Indian Ocean, op. cit., p. 216-222.

(37) Parmi les membres les plus réputés de l'Aligarh School il faut rappeler Irfan Habib, Satish Chandra, M. Athar Ali, Noman Siddiqi, S. Nurul Hasan, Iqtidar Alam Khan, Zahiruddin Malik et Shireen Moosvi. 
trouve le « centre » et où se trouve la « périphérie ${ }^{38}$, en raison tant du mercantilisme croissant de l'Angleterre et des Pays-Bas, que de la balance commerciale toujours défavorable pour l'Europe (qui a eu un constant écoulement d'argent pendant toute la première partie de l'âge moderne) $)^{39}$. Selon Paul Bairoch, par exemple, l'Inde possédait en 1750 24,8 \% du potentiel productif mondial, soutenu par un système commercial et bancaire très avancé $^{40}$. La crise successive de l'économie-monde indo-asiatique et son intégration dans le capitalisme mondial européen entre 1750 et 1850 n'est que la conséquence de la formalisation de la domination britannique au sortir de la bataille de Plassey $(1757)^{41}$.

Finalement, une dernière réponse a été formulée par les spécialistes de l'Asie du sud précoloniale (en particulier Chris Bayly, David Ludden, Peter Marshall et David Washbrook). Washbrook dénonce l'incapacité du modèle interprétatif du système-monde à capturer l'unicité de la région, car il ne considère pas le dynamisme historique qui caractérise les sociétés extra-européennes. La critique du modèle de Wallerstein - considéré comme une sorte d'orientalisme « renversé » - conduit à un nouveau point de vue

(38) Ravi PALAT et alii, « The Incorporation and Peripheralization of South Asia, 1600-1950 », Review, 10/1, 1986, p. 171-208. Pour le point de vue indo-centrique, voir Ashin DAS GUPTA, Indian Merchants and the Decline of Surat, c. 1700-1750, Wiesbaden, Steiner, 1979 ; Id., " India and the Indian Ocean in the Eighteenth century », dans Ashin DAS GUPTA, Michael N. PEARSON, India and the Indian Ocean, p. 132 ; Kirti N. CHAUdHURI, The World System, p. 226 ; Niels STEENSGAARD, Indian Ocean Network, p. 131, p. 133, p. 148-149; Michael PEARSON, Before colonialism, op.cit., p. 39-41 et 59-62 ; Om PRAKASH, European Commercial Enterprise, op. cit., p. 1, p. 8-9, p. 12-14, p. 22, p. 154, p. 163 et p. 186. Parmi les historiens indo-musulmans, voir Irfan HABIB, « The Eighteenth Century in Indian Economic History », dans Leonard BLUSSÉ, Femme GAASTRA (dir.), On the eighteenth Century as a Category of Asian History. Van Leur in Retrospect, Brookfield, Ashgate, 1998, p. 217-223 ; M. ATHAR ALI, «Recent Theories of Eighteenth-Century India », Indian Historical Review, 12/1 : 2, 1986-1987, p. 102-110 ; John RICHARDS, « Mughal State Finance and the Pre-modern World Economy », dans Comparative Studies in Society and History, 23/2, 1981, p. 296 ; Andre WINK, « Al-Hind : India and Indonesia in the Islamic World-Economy, c. 700-1800 AD », Itinerario, 12/1, 1988, p. 44-46, p. 54-55, p. 59 et p. 65-69 ; Peter MARShall (dir.), The Eighteenth Century in Indian History. Evolution or Revolution?, New Delhi, Oxford University Press, 2003.

(39) Voir Kirti N.CHAUDHURI, The Trading World of Asia and the English East India Company: 1660-1760, Cambridge, Cambridge University Press, 1978, p. 237, p. 153-160, p. 194, p. 197, p. 205, p. 215-220, p. 277-280 et p. 343-344 ; et Om PRAKASH, European Commercial Enterprise, op. cit., p. $337-339$.

(40) « The first world-market in manufactured goods» selon Paul BAIROCH («International industrialization levels from 1750-1980 », Journal of European Economic History, 11/2, 1982, p. 269333). Voir aussi David WASHBROOK, «From Comparative Sociology to Global History : Britain and India in the Pre-history of Modernity », dans Journal of the Economic and Social History of the Orient, 40/4, 1997, p. 416-418.

(41) Pour un point de vue critique à propos du modèle indo-centrique (en particulier pour une mise en discussion des perspectives se référant au système-monde et à l'économie-monde dans le scénario de l'Océan Indien), voir Remco RABEN, « The Broad Weft and Fragile Warp : Conference on the Eighteenth Century as a Category in Asian History », Itinerario, 18/1, 1994, p. 16. 
polycentrique, qui met en évidence le caractère multilatéral des relations entre les mondes ${ }^{42}$.

\section{Les frontières de l'Océan Indien}

En 1966 Braudel a posé avec urgence la question des frontières ${ }^{43}$ et, pour ce qui concerne l'Océan Indien en particulier, Chaudhuri a souligné que la question reste toujours ouverte et sans une réponse définitive ${ }^{44}$. De son côté, Bose a récemment écrit qu'on ne peut pas fournir une seule réponse à la question relative à la réelle extension géographique de l'Océan Indien $^{45}$. Et pourtant, parmi les objectifs de la nouvelle thalassologie, il y a autant la localisation que l'historicisation des études sur la région; mais ce courant s'attache également à des mots-clés plutôt indéterminés, tels que porosité, perméabilité, connectivité, flexibilité, ouverture des frontières et limites d'espace et de temps.

En ce qui concerne l'espace, une nouvelle théorie impose une prise de distance avec la géographie traditionnelle - qui s'attache aux valeurs, aux langages, aux pratiques matérielles et sociales, à l'écologie, etc., comme fondement d'une unité culturelle durable -, pour suivre l'exemple d'une autre géographie caractérisée par une attention particulière aux processus, aux modalités d'action, d'interaction et de mouvement (voyages, pèlerinages, trafics, guerres, prosélytisme, colonisation, exil, etc.) : selon ce point de vue, les régions peuvent être pensées de manière dynamique et interconnectée ${ }^{46}$.

Dans les dernières années, des nombreux auteurs ont porté leur attention sur des parcours de déterritorialisation, avec un soin particulier

(42) Voir David WASHBROOK, dans South Asia, op. cit., p. 58-59 ; Chris BAYLI, « Beating the Boundaries. South Asian History, c. 1700-1850 », dans Sugata BoSE (dir.), South Asia and World Capitalism, New Delhi-New York, Oxford University Press, 1990, p. 35-38 ; David LuDDEN, «World Economy and Village India, 1600-1900 : Exploring the Agrarian History of Capitalism», dans Sugata Bose, Ibidem, p. 159, p. 162-164 et p. 174-176 et Peter MARSHALL, « Retrospect on J.C. van Leur's Essay on the Eighteenth Century as a Category in Asian History », Itinerario, 17/1, 1993, p. 47.

(43) Fernand BRAUDEL, La Méditerranée et le monde méditerranéen à l'époque de Philippe II, Paris, Colin, 1966, vol. 1, p. 224. Voir aussi Kirti N. CHAUDHURI, Asia before Europe, op. cit., p. 112-113 ; Michael PEARSON, Indian Ocean, op. cit., p. 27-28 ; Sugata Bose, A Hundred Horizons. The Indian Ocean in the Age of Global Empire, p. 5.

(44) « How far the Indian Ocean made its influence felt in the vast sweep of land in the north and the south west, in the direction of Asia and Africa, is a fascinating question »: Kirti N. CHAUDHURI, Trade and civilisation, op. cit., p. 160.

(45) Sugata BoSE, A hundred Horizons. The Indian Ocean, p. 10.

(46) Voir David LUDDEN, «Area Studies in the Age of Globalisation», dans Frontiers, 6, 2000, p. 1-22 et Leila FAWAZ, Chris BAYLY (dir.), Modernity and Culture from the Mediterranean to the Indian Ocean, New York, Columbia University Press, 2002, p. 2-7. 
pour l'intégration, le mouvement et la migration : des constructions intellectuelles qui, une fois encore, posent en évidence le dualisme intégrationfragmentation qui est à la base des petites Méditerranées de l'Océan Indien $^{47}$

D'une façon plus concrète, la nouvelle thalassologie théorise un grand Océan Indien qui intègre les mers d'Asie (Frank Broeze), avec une série de systèmes régionaux étroitement interdépendants (qui s'étendent de l'Asie orientale à Afrique de l'est), aussi bien qu'avec les différentes sociétés côtières de l'océan (Michael Pearson). Quoique les frontières entre ces sociétés côtières et celles de l'intérieur du continent soient poreuses, flexibles et indéterminées, elles ont pourtant quelques éléments communs : le pluralisme, une prédisposition aux trafics sur la longue distance, l'alimentation, l'habitat, une vie liée au cycle des moussons, la diffusion d'une sorte de lingua franca et, finalement, une certaine religiosité populaire ${ }^{48}$.

Dans la même direction s'inscrit l'idée des cultures de frange, suggérée par l'anthropologue français Paul Ottino pour décrire les similitudes parmi les nombreuses sociétés syncrétiques qui se sont développées tout au long de la côte sud-est de l'Océan Indien, grâce au processus de métissage ${ }^{49}$. L'Océan Indien peut donc être considéré, suivant l'idée de Braudel sur la Méditerranée, comme un sujet constamment en expansion et en contraction,

(47) Arjun APPADURAI, Modernity at Large : Cultural Dimensions of Globalization, Minneapolis, University of Minnesota Press, 1996, p. 33 ; James ClifFORD, Routes. Travel and Translation in the Late Twentieth Century, Cambridge, Harvard University Press, 1997, p. 21, p. 25 et p. 36 ; Roland ROBERTSON, « Glocalization : time-Space and Homogeneity-Heterogeneity », dans Mike Featherstone, Scott LASH, Roland RoberTson (dir.), Global Modernities. From Hodernism to Hypermodernism and beyond, London, Sage Publications, 1995, p. 25-44 ; John R. MCNEILL, William H. MCNEILL, The Human Web. A Bird's-Eye View of World History, New York, W.W. Norton, 2003 ; Anthony REID, «Intra-Asian Networks : Global and Local in Southeast Asian History», International Journal of Asian Studies, 1/1, 2004, p. 5-6 ; David LUDDEN, « History outside Civilisation and the Mobility of South Asia », South Asia, 17/1, 1994, p. 7-8 ; Claude MARKOVITS, The Global World of Indian Merchants, 1750-1947. Traders of Sind from Bukhara to Panama, Cambridge-New York, Cambridge University Press, 2000 ; Claude MARKOVITS, Jean POUCHEPADASS, Sanjay SUBRAHMANYAM (dir.), Society and Circulation. Mobile People and Itinerant Cultures in South Asia, 1750-1950, London, Anthem, 2003.

(48) Voir Frank Broeze (dir.), Brides of the Sea. Port cities of Asia from the $16^{\text {th }}$-20th Centuries, Honolulu, University of Hawaii Press, 1989, p. 3 et p. 21 ; Michael PEARSON, «Littoral Society. A Case for the Coast», The Great Circle, 7/1, 1985, p. 1-9 (publié aussi dans Michael PEARSON, The World of the Indian Ocean, op. cit., p. 1-9) ; Id., « Littoral Society : The Concept and the Problems», Journal of World History, 17/4, 2006, p. 353-373. Voir aussi du même auteur, Indian Ocean, op. cit., p. 37-45.

(49) Paul OtTino, « La hiérarchie sociale et l'alliance dans le royaume de Mantacassi des XVI ${ }^{\mathrm{e}}$ et XVII ${ }^{\mathrm{e}}$ siècles », Tantara, 1/4, 1973, p. 53-89 (http://horizon.documentation.ird.fr/exldoc/pleins_textes/pleins_textes_5/b_fdi_06-07/07183.pdf). 
qui peut inclure ou exclure les régions voisines en raison des fluctuations économiques ${ }^{50}$.

D'autre côté, sur le plan de la périodisation on peut voir aussi une opération de déconstruction : à partir de la contraposition chronologique traditionnelle élaborée en Occident vis-à-vis de celle de l'Asie, on est passé à la construction d'une nouvelle vision temporelle eurasiatique (ou afro-eurasiatique), mieux capable d'intégrer les sujets et de les mettre en relation. Par rapport à l'histoire de l'Atlantique ou bien du Pacifique ${ }^{51}$, pour l'Océan Indien et son système-monde, il est vraiment difficile de trouver un accord sur une nouvelle périodisation partagée.

On trouve donc, à ce propos, des opinions totalement différentes, au point que le début d'un système-monde asiatique moderne a été situé par quelqu'un il y a 200 ans et, par d'autres, il y a $5000 \mathrm{ans}^{52}$. De toutes façons, nous pouvons retenir au moins quatre hypothèses principales dans le contexte d'un débat très vif sur le procès de modernisation et d'intégration économique de la région indienne.

Premièrement, l'Océan Indien peut-être considéré le plus ancien monde océanique et la première économie globale : la naissance précoce de trois grandes civilisations, dans le troisième millénaire avant J.C., dans la Mésopotamie, dans la vallée de l'Indus et autour des bouches du Nil a signé le début du processus de formation du premier système-monde. Et

(50) Fernand BRAUdel, La Méditerranée et le monde méditerranéen, op. cit., p. 168 ; Peregrine Horden, Nicholas PURCELL, «The "Mediterranean" and the New Thalassology», art. cit., p. 735. Pour le débat sur la world-economy dans l'Océan Indien et sur la périodisation, voir Fernand BRAUDEL, Civilisation matérielle, économie et capitalisme, op. cit., vol. III, p. 71-85; Kiri N. CHAUDHURI, Trading world, op. cit., p. 81-90 et 282-299.

(51) Voir Alfred CROSBY, The Columbian Exchange. Biological and Cultural Consequences of 1492, Westport, Greenwood Pub., 1973 ; John R. MCNEILL, «From Magellan to Miti : Pacific Rim Economies and Pacific Island Ecologies since $1521 »$, dans Sally MILLER, Anthony John H. LATHAM, Dennis FLYNN (dir.), Studies in the Economic History of the Pacific Rim, Routledge, London and New York 1998, p. 74.

(52) André GUNDER FRANK, «A theoretical Introduction to 5,000 Years of World System History », Review, 13/2, 1990, p. 155-248 ; André GUNDER FRANK, ReOrient : Global Economy in the Asian Age, Berkeley, University of California Press, 1998 ; Philippe BEAUJARD, «The Indian Ocean in Eurasian and African World-Systems before the Sixteenth Century», Journal of World History, 16/4, 2006, p. 420-459. Parmi les auteurs eurocentriques, voir David LANDES, The Wealth and Poverty of Nations. Why Some are so rich and Some so poor, New York, W.W. Norton, 1998 ; parmi les globalistes : Janet L. ABU-LuGHOD, Before European Hegemony. The World System A.D. 1250-1350; Pour un aperçu sur le débat, Jack GOLDSTONE, «Efflorescences and Economic Growth in World History : Rethinking the "Rise of the West" and the Industrial Revolution », Journal of World History, 13/2, 2002, p. 323-289 ; Jack GolDSTONE, « The Problem of the "Early Modern" World », Journal of the Economic and Social History of the Orient, 41/3, 1998, p. 249-284. 
le cœur de cet «Uruk world system », situé en Mésopotamie, a impliqué activement l'Océan Indien nord-occidental ${ }^{53}$.

Dans une deuxième hypothèse, l'intégration des espaces océaniques et côtiers de l'Océan Indien aurait commencé au moment où on a utilisé consciemment les moussons pour la navigation. À partir du troisième siècle avant J.C., on assiste donc à la naissance d'un grand réseau commercial indo-centrique, qui a permis la circulation soit des marchandises, soit des cultures et des religions (l'Hindouisme et le bouddhisme), et la formation d'une zone culturelle « macro-indienne $»^{54}$.

Un troisième point de vue se focalise sur la diffusion de l'Islam - à partir du VII ${ }^{\mathrm{e}}$ siècle - et sur la progressive constitution d'un réseau de centres mercantiles, politiques et religieux qui ont transformé l'Océan Indien en un « lac musulman ». À partir de la fin du $\mathrm{X}^{\mathrm{e}}$ siècle, ce réseau a commencé à s'étendre tout au long de trois directions - la mer d'Arabie, le Golfe du Bengale et la mer Chinoise du sud - et il a touché son zénith au début du XIV ${ }^{\mathrm{e}}$ siècle quand, grâce à une liaison continuelle avec les sociétés continentales (in primis la Chine), il s'est avéré fondamental pour la formation d'un «système-monde » capable de connecter l'Europe et l'Asie ${ }^{55}$.

Dans une quatrième hypothèse, l'arrivée des Européens dans l'Océan Indien à la fin du $\mathrm{XV}^{\mathrm{e}}$ siècle n'a pas signifié le début, ni d'une ère nouvelle caractérisée par une confrontation militaire et navale asymétrique (voir les opinions de Kavalam M. Panikkar, de Odayamadath Kunjappa Nambiar, de Bernard Vlekke et, avec des interprétations fort différentes, de Geoffrey Parker et de Carlo M. Cipolla) ${ }^{56}$, ni d'un «âge du partenariat » (Holden

(53) Voir Michael PeARson, Indian Ocean, op. cit., p. 49-50 ; Guillermo AlgAZE, The Uruk World System: the Dynamics of Expansion of Early Mesopotamian civilization, Chicago, University of Chicago Press, 2004 ; Shereen RATNAGAR, Trading Encounters. From the Euphrates to the Indus in the Bronze Age, New Delhi, Oxford University Press, 2004.

(54) Lynda SHAFFER, « Southernization », Journal of World History, 5/1, 1994, p. 1 et p. 4. Pour un point de vue critique, voir John VoLL, «Southernization as a Construct in post-civilizational Narrative», dans Ross DUNN (dir.), The New World History. A Teacher's Companion, Boston-New York, Bedford and St. Martin's, 2000, p. 193-195.

(55) Patricia RISSO, Merchants and Faith : Muslim Commerce and Culture in the Indian Ocean, Boulder, Westview Press, 1995 ; John VoLL, «Islam as a special World System», dans Journal of World History, 5/2, 1994, p. 213-226 ; Richard EATON, « Islamic History as World History », dans Richard EATON, Essays on Islam and Indian History, New Delhi-Oxford, Oxford University Press, 2000, p. 9-44 ; Ashin DAS GUPTA, Michael PEARSON, India and the Indian Ocean, op. cit., p. 11-20.

(56) Kavalam M. PANIKKAR, Asia and Western Dominance. A Survey of the Vasco da Gama Epoch of Asian History, 1498-1 945, London, Allen \& Unwin, 1959, p. 13-15 ; Clifford RoGERS (dir.), The Military Revolution Debate : Readings on the Military Transformation of Early Modern Europe, Boulder, Westview Press, 1995 ; Geoffrey PARKER, The Military Revolution. Military Innovation and the Rise of the West, 1500-1800, Cambridge, Cambridge University Press, 1996 (2e éd.) ; Carlo M. CIPOLla, Guns, Sails, and Empires : Technological Innovation and the Early Phases of European 
Furber), ou bien d'un «âge du commerce » (Anthony Reid, Kenneth McPherson) ou, finalement, d'un « âge de la réciprocité » (Michael Pearson) ou de la « convivialité » (Anthony John R. Russell-Wood) ${ }^{57}$. Suivant cette hypothèse, l'expansion européenne, au contraire, a été à l'origine d'un «âge du conflit retenu » (Sanjay Subrahmanyam), d'un « équilibre du chantage » (Ashin Das Gupta), d'une «perception de l'avantage mutuel » (Om Prakash), d'une « synthèse qui dépasse les conflits » (Chris Bayly), d'une « coopération résignée » et d' « accommodements »(Peter Marshall), ou encore - selon David Ludden - de « double dépendance $»^{58}$. On peut donc parler d'une époque tant de conflits que de coopération, où guerre et commerce ont avancé ensemble, une époque aussi où les Européens étaient obligés de s'adapter aux structures locales préexistantes.

Ainsi, l'époque de la première globalisation ${ }^{59}$ a vu le début de l'intégration de l'univers complexe de l'Océan Indien dans un monde plus vaste, même si les liens restèrent faibles et généralement sans conflictualité particulière. Les signaux d'un changement imminent furent : la diffusion du christianisme, l'utilisation d'une lingua franca (c'est-àdire le portugais et le portugais-créole), la naissance d'une communauté Eurasiatique (en particulier les Indo-portugais), la création des nouvelles villes portuaires gouvernées par les Européens (Goa, Manille, Batavia, Pondichéry, Calcutta, etc.), le volume croissant du commerce européen à travers le Cap de Bonne Espérance et, facteur plus important, l'arrivée

Expansion, 1400-1700, New York, Minerva Press, 1965, p. 138-146 ; Peter MARSHALL, « Western Arms in Maritime Asia in the Early Phases of Expansion », Modern Asian Studies, 14/1, 1980, p. 13-28.

(57) Voir: Holden FURBER, Rival empires of trade in the Orient, 1600-1800, Minneapolis, University of Minnesota Press, 1976, p. 324 ; Id., John Company at Work. A Study of European Expansion in India in the Late Eighteenth Century, Cambridge, Harvard University Press, 1948 ; Id., «Asia and the West as Partners before "Empire" and after», Journal of Asian Studies, 28/4, 1969, p. 711-721 ; Anthony REID, «An "Age of Commerce" in Southeast Asian history », Modern Asian Studies, 24/1, 1990, p. 1-30 ; Michael PEARson, The Portuguese in India, Cambridge, Cambridge University Press, 1987, p. 2, p. 61, p. 81, p. 103-106 et p. 115 ; Anthony John R. RUSSELL-WOOD, The Portuguese Empire, 1415 -1808: a World on the Move, Baltimore, Johns Hopkins University Press, 1998, p. XXI, p. 21 et p. 220.

(58) Ashin DAS GUPTA, Europeans in India before the Empire, dans Ashin DAS GUPTA, Indian Ocean Merchant, op.cit., p. 229-230 ; Om PRAKASH, « European Corporate Enterprises and the Politics of Trade in India, 1600-1800 », dans Rudrangshu MUKHERJEE, Lakshmi SUBRAMANIAN, Politics and Trade, Oxford, Oxford University Press, 2003, op. cit., p. 173-174. Voir aussi Chris BAYLY, The Birth of the Modern World, 1780-1914. Global Connections and Comparisons, Malden, Blackwell, 2004, p. 476.

(59) Geoffrey GunN, First Globalization : the Eurasian Exchange, 1500-1800, Lanham, Rowman \& Littlefield, 2003 ; Chris BAYLY, «"Archaic" and "Modern" globalization in the Eurasian and African Arena», dans Anthony HOPKINS (dir.), Globalization in World History, New York, Norton, 2002, p. 47-73 ; Chris BAYLY, «From Archaic Globalization to International Networks, circa 16002000 », dans Jerry BENTLEY, Renate BRIDENTHAL, Anand YANG (dir.), Interactions. Transregional Perspectives on World History, Honolulu, University of Hawaii Press, 2005, p. 14-29. 
massive de l'argent d'Amérique qui détermina l'intégration progressive du système-monde asiatique dans le système américain et interocéanique. La première globalisation européenne était déjà commencée ${ }^{60}$.

Toutefois c'est le $\mathrm{XIX}^{\mathrm{e}}$ siècle qui a signé une vraie révolution systémique dans l'économie-monde asiatique : les transformations déterminées par le colonialisme et par le capitalisme industriel ont produit des changements politiques et économiques définitifs, qui ont fermé un cycle de l'histoire asiatique. Selon Michael Pearson, c'est à cette époque qu'on peut situer le tournant majeur d'une histoire de l'Océan Indien (toute à l'intérieur d'un univers géopolitique spécifique) à une histoire dans l'Océan Indien, influencée par des dynamiques externes à la région : une interprétation partagée par la plupart des chercheurs spécialistes de cette zone géopolitique et par le courant de la nouvelle thalassologie ${ }^{61}$.

D'ailleurs, quelques auteurs, tel que Sugata Bose, ont critiqué une adhésion sans conditions à la théorie qui voit une dissolution de la spécificité de la zone géopolitique de l'Océan Indien dans un monde où les distances sont annulées par des processus d'intégration systémique, en soulignant que, même après 1830, l'Océan Indien a eu une importance considérable comme zone intermédiaire entre les niveaux de la nation et du globe ${ }^{62}$.

D'autre part le débat sur le début de la modernité est encore ouvert : Chris Bayly, par exemple, a écrit que si le « long »XIX ${ }^{\mathrm{e}}$ siècle (1780-1914) a sans doute signé la naissance du monde développé, le «long » XVIII siècle (1680-1820) a toutefois contribué à sa naissance, même avec de nombreux doutes et contradictions. Jack Goldstone et d'autres chercheurs de la California school ont nié la possibilité de parler d'une croissance économique du monde moderne avant le $\mathrm{XIX}^{\mathrm{e}}$ siècle $^{63}$, et finalement Burton Stein et Irfan Habib ont refusé l'idée d'un nouvel âge moghol, revenant

(60) Dennis FlynN, Arturo GIRÁLDEZ, « Cycles of Silver : global Economic Unity through the Mid-Eighteenth Century », Journal of World History, 13/2, 2002, p. 391-427.

(61) Michael PEARSON, Indian Ocean, op; cit., chap. 7 et 8. Voir aussi Ashin DAS GuPTA, Michael PEARSON, India and the Indian Ocean, op. cit., p. 39 ; Kiri N. CHAUDHURI, Trade and Civilization, p. 211 ; Kenneth MCPHERSON, Indian Ocean, op. cit., p. 198-200 ; André WINK, « AlHind : India and Indonesia in the Islamic World-Economy, c. 700-1800 AD », Itinerario, vol. XII, 1988 , p. 3.

(62) «Le défi à ce propos est de maintenir comme une unité analytique la zone interrégionale d'interaction culturelle et économique de l'Océan Indien, en évitant en même temps les risques qui découlent du postulat d'une quelconque thèse, simpliste et insoutenable, établissant la continuité»: Sugala Bose, A Hundred Horizons. The Indian Ocean in the Age of Global Empire, op. cit., p. 20-21.

(63) Voir Chris A. BAYLY, The Birth of the Modern World, 1780-1914, Oxford, Oxford Blackwell, 2004 ; Jack. A. GoLDSTONE, «Efflorescences and Economic Growth in World History : Rethinking the "Rise of the West" and the Industrial Revolution » art.cit; et id., « The Problem of the "Early Modern" world », Journal of the Economic and Social History of the Orient, vol. 41, n³, 1998. 
à une définition «classique » de fin du Moyen Âge pour l'histoire de la région entre $\mathrm{XVIII}^{\mathrm{e}}$ et $\mathrm{XIX}^{\mathrm{e}}$ siècle ${ }^{64}$.

\section{Potentialités et dangers de la nouvelle thalassologie}

Du point de vue de l'historiographie, quels sont donc les résultats obtenus par le travail de ces nouvelles écoles?

Sans doute, une perspective qui a mis au centre l'océan a-t-elle permis d'éliminer plusieurs distinctions artificielles entre zones géographiques traditionnellement présentées comme séparées l'une de l'autre (Asie, Afrique, Europe, etc.) et, en même temps, a porté l'attention sur les relations systémiques et de longue durée qui ont lié ensemble, par la médiation de l'Océan Indien et de ses «petites Méditerranées », des régions différentes. Historiciser l'océan a permis de mettre en évidence des processus historiques de longue durée et de comprendre la dimension des échanges commerciaux, biologiques et culturels entre différentes régions ${ }^{65}$.

D'ailleurs les espaces maritimes sont des réalités intrinsèquement instables. Les frontières et les caractéristiques des régions côtières peuvent dramatiquement changer, ainsi que les relations entre les mers et les continents : les interactions globales ont miné la cohérence des différentes régions maritimes et ont rendu encore plus évident le problème de la définition des frontières spatiales ${ }^{66}$.

Avant la globalisation de la période moderne et la « mort » de l'espace (entendu comme distance), les régions maritimes ont été généralement représentées comme des mondes séparés et fragmentés : Sugata Bose a justement observé que les sociétés qui bordent l'Océan Indien ont «néanmoins eu des frontières internes et externes flexibles ${ }^{67}$.

La nouvelle thalassologie, donc, peut être définie comme l'histoire, dans le sens le plus large, de la création, de la destruction et de la

(64) Burton STEIN, A history of India, Oxford, Blackwell, 1998, p. 198-200.

(65) Bernhard KLEIN, Gesa MACKENTHUN (dir.), Sea changes. Historicizing the Ocean, New York, Routledge, 2004, p. 1-12.

(66) «Selon ce point de vue, les efforts pour construire des bassins océaniques et maritimes séparés comme de grandes aires géographiques, à l'époque moderne, peuvent même apparaître comme une nouvelle version du mythe des continents, en vertu duquel le monde atlantique signifierait une «Grande Europe », l'Océan Indien représenterait une "Grande Asie », et le bassin du Pacifique apparaîtrait comme un terrain de jeu exotique pour les hardis baleiniers et les explorateurs européens, repoussés ensuite par les capitalistes euro-américains et d'Asie orientale »: Jerry BENTLEY, « Sea and Ocean Basins as Frameworks of Historical Analysis », Geographical Review, 89/2, 1999, p. 215-224.

(67) Sugata BOSE, « Space and Time on the Indian Ocean Rim : theory and History », Leila Tarazi FAWAZ, Chris A. BAYLY, Modernity and Culture from the Mediterranean to the Indian Ocean, p. 368-369 ; Id., A Hundred Horizons. The Indian Ocean in the Age of Global Empire, op. cit., p. 6. 
recréation de communautés, en raison du mouvement (des personnes, des marchandises, des pratiques culturelles et des idées) à travers et autour de l'Océan Indien.

Suivant les réflexions élaborées par John Elliott à propos de l'Atlantique, on peut donc soutenir que l'Océan Indien aussi devrait être représenté à la fois comme un élément fédérateur, reliant les peuples et les événements à travers l'océan, et comme un élément de division, de la fragmentation et de l'éloignement des communautés. Il s'agit d'une « histoire dans laquelle les paradoxes abondent - l'intégration et la fragmentation, les liens qui nous unissent et les forces qui divisent $»^{68}$.

Silvia M. PIZZETTI

Université de Milan silvia.pizzetti@unimi.it Traduction assurée par l'auteure p. 249. 